\title{
203. On the Stability of Solutions of Some Non-Autonomous Differential Equations of the Third Order
}

\author{
By Minoru Yamamoto \\ Osaka University \\ (Comm. by Kenjiro ShodA, M. J. A., Sept. 13, 1971)
}

1. Introduction. In this note we investigate the asymptotic stability in the large, as $t \rightarrow \infty$, of the zero solution of the differential equation

$$
\dddot{x}+\psi(t, x, \dot{x}, \ddot{x})+\phi(t, x, \dot{x})+c(t) f(x)=0,
$$

where $\psi, \phi, f$ and $c$ are real valued functions. The dots indicate differentiation with respect to $t$ and all solutions considered are assumed real.

In [4] K. E. Swick established conditions under which all solutions of the non-autonomous equations

$$
\begin{gathered}
\dddot{x}+p(t) \ddot{x}+q(t) g(\dot{x})+r(t) h(x)=0 \\
\dddot{x}+f(t, x, \dot{x}) \ddot{x}+q(t) g(\dot{x})+r(t) h(x)=0
\end{gathered}
$$

tend to the zero solution as $t \rightarrow \infty$.

Recently, in [2] T. Hara also obtained some conditions under which all solutions of the equation

$$
\begin{gathered}
\dddot{x}+a(t) \ddot{x}+b(t) \dot{x}+c(t) x=0 \\
\dddot{x}+a(t) f(x, \dot{x}) \ddot{x}+b(t) g(x, \dot{x}) \dot{x}+c(t) x=0
\end{gathered}
$$

tend to the zero solution as $t \rightarrow \infty$.

To prove the following theorems (see 3) we construct a Liapunov function using the well-known techniques which are frequent in [3].

2. Auxiliary Lemmas, Consider the sytem of differential equation

$$
\dot{X}=\boldsymbol{F}(t, X)
$$

where $X=\left(x_{1}, \cdots, x_{n}\right), F(t, 0)=0$ for $t \in I=[0,+\infty)$ and $F(t, X)$ is continuous in $I \times R^{n}$.

The following lemmas are well-known and play the essential role to prove the theorems, see [5] (Th. 8.1, Th. 10.2, Th. 14.2).

Lemma 2.1. Suppose that there exists a Liapunov function $V(t, X)$ defined on $0 \leqq t<\infty,\|X\|<H(H>0)$, which satisfies the following conditions; (i) $V(t, 0) \equiv 0$, (ii) $w_{1}(\|X\|) \leqq V(t, X)$, where $w_{1}(r)$ is a continuous increasing, positive definite function, (iii) $\dot{V}_{(2.1)}(t, X) \leqq 0$. Then, the zero solution $X(t) \equiv 0$ of the system (2.1) is stable.

Lemma 2.2. Suppose that there exists a Liapunov function $V(t, X)$ 
defined on $0 \leqq t<+\infty,\|X\| \geqq R$ where $R$ may be large, which satisfies the following conditions (i) $w_{1}(\|X\|) \leqq V(t, X) \leqq w_{2}(\|X\|)$ where $w_{1}(r)$ and $w_{2}(r)$ are continuous increasing functions such that $w_{1}(r) \rightarrow \infty$ as $r \rightarrow \infty$. (ii) $\dot{V}_{(2.1)}(t, X) \leqq 0$. Then, the solutions of (2.1) are uniform-bounded.

Lemma 2.3. Suppose that there exists a non-negative Liapunov function $V(t, X)$ defined in $I \times R^{n}$ such that $\dot{V}_{(2.1)}(t, X) \leqq-w_{3}(X)$, where $w_{3}(X)$ is positive definite with respect to a closed set $\Omega$ in the space $R^{n}$. Moreover, suppose that $F(t, X)$ is bounded for all $t$ when $X$ belongs to an arbitrary compact set in $R^{n}$ and that there exists a function $H(X)$ defined on $\Omega$ such that:

(a) $F(t, X)$ tends to $H(X)$ for $X \in \Omega$ as $t \rightarrow \infty$ and on any compact set in $\Omega$ this covergence is uniform.

(b) Corresponding to each $\varepsilon>0$ and each $Y \in \Omega$, there exist $a$ $\delta(\varepsilon, Y)>0$ and $a T(\varepsilon, Y)>0$ such that if $\|X-Y\|<\delta(\varepsilon, Y)$ aud $t>T(\varepsilon, Y)$ we have $\|\boldsymbol{F}(t, X)-\boldsymbol{F}(t, Y)\|<\varepsilon$.

Then, every bounded solution of (2.1) approaches the largest semiinvariant set of the system $\dot{X}=H(X)$ contained in $\Omega$ as $t \rightarrow \infty$. In particular, if all the solutions of (2.1) are bounded, every solution of (2.1) approaches the largest semi-invariant set of $\dot{X}=H(X)$ contained in $\Omega$ as $t \rightarrow \infty$.

3. Assumptions and Theorems. We now state the assumptions on the functions $\psi, \phi, f$ and $c$ appeared in the equation (1.1).

Assumptions. ( I ) $f(x)$ is a continuously differentiable function in $R^{1}$, and $c(t)$ is continuously differentiable in $I=[0, \infty)$.

(II ) The function $\phi(t, x, y)$ is continuous in $I \times R^{2}$. For the function $\phi$ there exist functions $b(t), \phi_{0}(x, y)$ and $\phi_{1}(x, y)$ which satisfy the inequalities

$$
b(t) \phi_{0}(x, y) \leqq \phi(t, x, y) \leqq b(t) \phi_{1}(x, y)
$$

for all $(t, x, y) \in I \times R^{2}$. The function $b(t)$ is continuously differentiable in $I$, and functions $\phi_{0}(x, y), \phi_{1}(x, y), \frac{\partial \phi_{0}}{\partial x}(x, y)$ and $\frac{\partial \phi_{1}}{\partial x}(x, y)$ are continuous in $R^{2}$.

(III) The function $\psi(t, x, y, z)$ is continuous in $I \times R^{3}$. For the function $\psi$ there exist functions $a(t), \psi_{0}(x, y)$ and $\psi_{1}(x, y)$ which satisfy the inequalities

$$
a(t) \psi_{0}(x, y) z \leqq \psi(t, x, y, z) \leqq a(t) \psi_{1}(x, y) z
$$

for all $(t, x, y, z) \in I \times R^{3}$. Further the function $a(t)$ is continuously differentiable in $I$, and functions $\psi_{0}(x, y), \psi_{1}(x, y), \frac{\partial \psi_{0}}{\partial x}(x, y)$ and $\frac{\partial \psi_{1}}{\partial x}(x, y)$ are continuous in $R^{2}$. The following notations are also used:

$$
\widetilde{\phi}(x, y)=\frac{1}{2}\left\{\phi_{0}(x, y)+\phi_{1}(x, y)\right\}, \quad \widetilde{\psi}(x, y)=\frac{1}{2}\left\{\psi_{0}(x, y)+\psi_{1}(x, y)\right\} .
$$


Theorem 1. Suppose that the assumptions (I), (II) and (III) above and that these functions satisfy the following conditions:

(1) $f(0)=0, \frac{f(x)}{x} \geqq f_{0}>0 \quad(x \neq 0)$

(2) $f^{\prime}(x) \leqq f_{1} \leqq 1$

(3) $\phi(t, x, 0)=0 \quad$ in $I \times R$ $\frac{\widetilde{\phi}(x, y)}{y} \geqq \phi_{0}>0 \quad(y \neq 0), \quad \tilde{\phi}_{x}(x, y) \leqq 0 \quad$ in $R^{2}$

(4) $\tilde{\psi}(x, y) \geqq \tilde{\psi}_{0}>0, \quad \tilde{\psi}_{x}(x, y) y \leqq 0 \quad$ in $R^{2}$

( 5 ) $\quad 0<c_{0} \leqq c(t) \leqq c_{1}, \quad 0<b_{0} \leqq b(t) \leqq b_{1}, \quad 0<a_{0} \leqq a(t) \leqq a_{1}$

(6) $\sup _{y \neq 0} \frac{1}{y}\left\{\phi_{1}(x, y)-\phi_{0}(x, y)\right\}=p_{1}$ $\sup \left\{\psi_{1}(x, y)-\psi_{0}(x, y)\right\}=q_{1}$

( 7 ) $a_{0} b_{0} \phi_{0} \psi_{0}>c_{1}$

( 8 ) $8\left(\mu b_{0} \phi_{0}-c_{1} f_{1}\right)\left(a_{0} \psi_{0}-\mu\right)>\left(\mu a_{1} q_{1}+b_{1} p_{1}\right)^{2}$ where $\mu=\frac{a_{0} b_{0} \phi_{0} \psi_{0}+c_{1}}{2 b_{0} \phi_{0}}$

(9) $\sup _{t \in I}\left[\mu^{2}\left|\frac{a^{\prime}(\mathrm{t})}{a_{0}}\right|+\frac{1}{\mu}\left\{\left|\frac{b^{\prime}(t)}{b_{0}}\right| c(t)-c^{\prime}(t)\right\}\right]<\frac{a_{0} b_{0} \phi_{0} \psi_{0}-c_{1}}{2}$

$$
\begin{aligned}
& \int_{0}^{\infty}\left|a^{\prime}(t)\right| d t<+\infty, \int_{0}^{\infty}\left|b^{\prime}(t)\right| d t<+\infty, \int_{0}^{\infty}\left|c^{\prime}(t)\right| d t<+\infty \text { and } \\
& c^{\prime}(t) \rightarrow 0 \quad \text { as } t \rightarrow \infty .
\end{aligned}
$$

Then every solution of (1.1) is uniform-bounded and satisfies $x(t) \rightarrow 0$, $\dot{x}(t) \rightarrow 0, \ddot{x}(t) \rightarrow 0$ as $t \rightarrow \infty$.

Next, considering the equation

$$
\dddot{x}+a(t) \psi(x, \dot{x}) \ddot{x}+b(t) \phi(x, \dot{x})+c(t) f(x)=0
$$

we can take the function $\phi(x, y)$ in place of $\phi_{0}(x, y)$ and $\phi_{1}(x, y)$, and the function $\psi(x, y)$ in place of $\psi_{0}(x, y)$ and $\psi_{1}(x, y)$ in the Assumptions (II), (III). Thus in this case the functions $\tilde{\phi}(x, y)$ and $\tilde{\psi}(x, y)$ coincide with $\phi(x, y)$ and $\psi(x, y)$ respectively, and $p_{1}=q_{1}=0$. Thus from the Theorem 1 , we have

Theorem 2. Suppose that the functions $a(t), b(t)$ and $c(t)$ are continuously differentiable in $I=[0,+\infty)$, and the functions $f(x)$, $\phi(x, y), \psi(x, y), \phi_{x}(x, y)$ and $\psi_{x}(x, y)$ are continuous in $R^{2}$, and that these functions satisfy the following conditions:

(1) $\quad f(0)=0 \quad \frac{f(x)}{x} \geqq f_{0}>0 \quad(x \neq 0), f^{\prime}(x) \leqq 1$

( 2) $\phi(x, 0)=0$

$$
\frac{\phi(x, y)}{y} \geqq \phi_{0}>0 \quad(y \neq 0), \phi_{x}(x, y) \leqq 0 \text { in } R^{2}
$$

(3) $\psi(x, y) \geqq \psi_{0}>0, \quad \psi_{x}(x, y) y \leqq 0 \quad$ in $R^{2}$ 
(4) $0<c_{0} \leqq c(t) \leqq c_{1}, \quad 0<b_{0} \leqq b(t) \leqq b_{1}, \quad 0<a_{0} \leqq a(t) \leqq a_{1}(t \in I)$

( 5 ) $\quad a_{0} b_{0} \phi_{0} \psi_{0}>c_{1}$

(6) $\sup _{t \in I}\left[\mu^{2}\left|\frac{a^{\prime}(t)}{a_{0}}\right|+\frac{1}{\mu}\left\{\left|\frac{b^{\prime}(t)}{b_{0}}\right| c(t)-c^{\prime}(t)\right\}\right]<\frac{a_{0} b_{0} \phi_{0} \psi_{0}-c_{1}}{2}$ where

$$
\mu=\frac{a_{0} b_{0} \phi_{0} \psi_{0}+c_{1}}{2 b_{0} \phi_{0}}
$$

$$
\begin{aligned}
& \int_{0}^{\infty}\left|a^{\prime}(t)\right| d t<+\infty, \int_{0}^{\infty}\left|b^{\prime}(t)\right| d t<+\infty, \int_{0}^{\infty}\left|c^{\prime}(t)\right| d t<+\infty \text { and } \\
& c^{\prime}(t) \rightarrow 0 \text { as } t \rightarrow \infty .
\end{aligned}
$$

Then every solution of (3.1) is uniform-bounded and satisfies $x(t)$ $\rightarrow 0, \dot{x}(t) \rightarrow 0, \ddot{x}(t) \rightarrow 0$ as $t \rightarrow \infty$.

Remark. In the case that the equation (3.1) reduces to an autonomous equation, that is, the functions $a(t), b(t)$ and $c(t)$ are independent of $t$, the conditions (6), (7) of the Theorem 2 are automatically satisfied, and our results coincide with that obtained by J. O. C. Ezeilo [1] and so forth. Especially when the equation (3.1) reduces to a linear differential equation with constant coefficients, then the conditions described in the Theorem 2 coincide with the Routh-Hurwitz condition.

4. Proof of Theorem 1. First of all we note that the equation (1.1) is equivalent to the following system of differential equations

(4.1) $\quad \dot{x}=y, \quad \dot{y}=z, \quad \dot{z}=-f(t, x)-\phi(t, x, y)-\psi(t, x, y, z)$.

A Liapunov function satisfying the conditions assumed in the auxiliary lemmas will be given as follows ;

$$
\begin{aligned}
U(t, x, y, z) & \\
= & e^{-\int_{0}^{t} \rho(t) d t} V(t, x, y, z) \text {, where } \rho(t)=\left|\frac{a^{\prime}(t)}{a_{0}}\right|+\left|\frac{b^{\prime}(t)}{b_{0}}\right|+\left|\frac{c^{\prime}(t)}{c_{0}}\right| \\
2 V(t, x, y, z) & \\
= & 2 \mu c(t) \cdot \int_{0}^{x} f(\xi) d \xi+2 c(t) f(x) y+2 b(t) \int_{0}^{y} \tilde{\phi}(x, \eta) d \eta \\
& +2 \mu a(t) \cdot \int_{0}^{y} \widetilde{\psi}(x, \eta) \eta d \eta+2 \mu y z+z^{2} \\
= & 2 \mu c(t) \int_{0}^{x}\left\{1-f^{\prime}(\xi)\right\} f(\xi) d \xi+\mu c(t)\left\{f(x)+\frac{y}{\mu}\right\}^{2} \\
& +(\mu y+z)^{2}+\frac{2}{\mu} \int_{0}^{y}\{\mu b(t) \tilde{\phi}(x, \eta)-c(t) \eta\} d \eta \\
& +2 \mu \int_{0}^{y}\{a \tilde{\psi}(x, \eta)-\eta\} d \eta .
\end{aligned}
$$

From the condition $a_{0} \psi_{0}>\mu>\frac{c_{1}}{b_{0} \phi_{0}}$ there exists a positive number $\delta$ such that $\mu(1-\delta) b_{0} \phi_{0}>c_{1}$ and $a_{0} \psi_{0}(1-\delta)>\mu$.

Let $\delta_{0}=\min \left\{\mu c_{0} \delta f_{0}^{2}, b_{0} \phi_{0}-\frac{c_{1}}{\mu(1-\delta)}+\mu a_{0} \psi_{0}-\frac{\mu^{2}}{(1-\delta)^{2}}, \delta\right\}$, then we easily have for $t \in I$, 


$$
U(t, x, y, z) \geqq e^{-\int_{0}^{\infty} \rho(t) d t} \cdot \delta_{0} \cdot\left(x^{2}+y^{2}+z^{2}\right)=w_{1}(\|x\|) .
$$

The existence of continuous increasing function $w_{2}(\|X\|),(\|X\|$ $\left.=\sqrt{x^{2}+y^{2}+z^{2}}\right)$ satisfying

$$
U(t, x, y, z) \leqq w_{2}(\|X\|)
$$

is easily obtained, and we have

$$
\begin{aligned}
& e^{-\int_{0^{\prime}(t) d t}^{t}} \dot{U}_{(4.1)}(t, x, y, z) \\
&=\dot{V}_{(4.1)}(t, x, y, z)-\rho(t) V(t, x, y, z) \\
&=-2\left\{\mu \phi(t, x, y)-c(t) f^{\prime}(x) y\right\} y-2\{\psi(t, x, y, z)-\mu z\} z \\
&-2\left\{c(t) \rho(t)-c^{\prime}(t)\right\} \cdot \int_{0}^{x}\left\{1-f^{\prime}(\xi)\right\} f(\xi) d \xi \\
&+\frac{1}{\mu}\left\{\left|\frac{b^{\prime}(t)}{b_{0}}\right| c(t)-c^{\prime}(t)\right\} y^{2}-2\left\{\left|\frac{b^{\prime}(t)}{b_{0}}\right| b(t)-b^{\prime}(t)\right\} \cdot \int_{0}^{y} \tilde{\phi}(x, \eta) d \eta \\
&-\frac{2}{\mu}\left\{\left|\frac{a^{\prime}(t)}{a_{0}}\right|+\left|\frac{c^{\prime}(t)}{c_{0}}\right|\right\} \int_{0}^{y}\{\mu b(t) \phi(x, \eta)-c(t) \eta\} d \eta \\
&-2 \mu\left\{\left|\frac{b^{\prime}(t)}{b_{0}}\right|+\left|\frac{c^{\prime}(t)}{c_{0}}\right|\right\} \cdot \int_{0}^{y}\{a(t) \psi(x, \eta)-\mu\} \eta d \eta \\
&-2 \mu\left\{\left|\frac{a^{\prime}(t)}{a_{0}}\right| a(t)-a^{\prime}(t)\right\} \cdot \int_{0}^{y} \tilde{\psi}(x, \eta) \eta d \eta+\mu^{2}\left|\frac{a^{\prime}(t)}{a_{0}}\right| y^{2} \\
&-\mu\left\{\rho(t) c(t)-c^{\prime}(t)\right\}\left\{f(x)+\frac{y}{\mu}\right\}^{2}-\rho(t)(\mu y+z)^{2} \\
&+2\{b(t) \tilde{\phi}(x, y)-\phi(t, x, y)\} z+2 \mu\{a(t) \tilde{\psi}(x, y) z-\tilde{\psi}(t, x, y, z)\} y \\
& \leqq-\left[\left(\mu b_{0} \phi_{0}-c_{1} f_{1}\right)-\left\{\mu^{2}\left|\frac{a^{\prime}(t)}{a_{0}}\right|+\frac{1}{\mu}\left(\left|\frac{b^{\prime}(t)}{b_{0}}\right| c(t)-c^{\prime}(t)\right)\right\}\right] y^{2} \\
&-\left(a_{0} \psi \psi_{0}-\mu\right) z^{2} \leqq-\delta_{1}\left(y^{2}+z^{2}\right)
\end{aligned}
$$

for suitable choice of positive $\delta_{1}$. Therefore we have

$$
\dot{U}_{(4.1)}(t, x, y, z) \leqq-\delta_{1} \cdot\left(y^{2}+z^{2}\right) e^{-\int_{0}^{\infty} \rho(t) d t}=w_{3}(x, y, z)
$$

and (4.4), (4.5), (4.6), Lemma 2.1 and Lemma 2.2 show that the zero solution of (4.1) is stable and all the solutions of (4.1) are uniformbounded.

The function $w_{3}(x, y, z)$ is positive definite with respect to the closed set $\Omega=\left\{(x, y, z) \mid x \in R^{1}, y=0, z=0\right\}$.

Let

$$
F(t, x, y, z)=\left(\begin{array}{c}
y \\
z \\
-c(t) f(x)-\phi(t, x, y)-\psi(t, x, y, z)
\end{array}\right),
$$

then $F(t, x, y, z)$ is bounded for all $t \in I$ when $(x, y, z)$ belongs to an arbitrary compact set in $R^{3}$, and $F(t, x, y, z)=\left(\begin{array}{c}0 \\ 0 \\ -c(t) f(x)\end{array}\right)$ in $\Omega$, and $c(t) \rightarrow c_{\infty}$ as $t \rightarrow \infty\left(c_{0} \leqq c_{\infty} \leqq c_{1}\right)$.

If we set 


$$
H(x, y, z)=\left(\begin{array}{c}
0 \\
0 \\
-c_{\infty} f(x)
\end{array}\right),
$$

then conditions (a) and (b) in Lemma 2.3 are all satisfied, and since all solutions of (4.1) are bounded as obtained above, it follows that every solution of (4.1) approaches the largest semi-invariant set of the system $\dot{X}=H(X)$ contained in $\Omega$ as $t \rightarrow \infty$. The system $\dot{X}=H(X)$ is a system of equations

and has the general solution

$$
\dot{x}=0, \quad \dot{y}=0, \quad \dot{z}=-c_{\infty} \cdot f(x)
$$

$$
x=\gamma_{1}, \quad y=\gamma_{2}, \quad z=\gamma_{3}-c_{\infty} \cdot f\left(\gamma_{1}\right) t .
$$

In order to remain in $\Omega$, the above solutions must satisfy the conditions : $\gamma_{2}=0$ and $\gamma_{3}-c_{\infty} \cdot f\left(\gamma_{1}\right) t=0$ for all $t \geqq 0$, and these imply $\gamma_{3}=0$, $f\left(\gamma_{1}\right)=0$, and thus $\gamma_{3}=0, \gamma_{1}=0$. Therefore the only solution of $\dot{X}$ $=H(X)$ remained in $\Omega$ is $X=0$, that is, the largest semi-invariant set of the system $\dot{X}=H(X)$ contained in $\Omega$ coincides with the point $(0,0,0)$. Thus we have the conclusion from Lemma 2.3.

Details are somewhat complicated and will be published later.

\section{References}

[1] J. O. C. Ezeilo: On the stability of solutions of certain differential equations of the third order. Quart. J. Math., 11(2), 64-69 (1960).

[2] T. Hara: On the stability of solutions of certain third order ordinary differential equation. Proc. Japan Acad., 47, 897-902 (1971).

[ 3 ] R. Reissig, G. Sansone und R. Conti: Nichtlineare Differentialgleichungen Höherer Ordnung. Roma (1969).

[4] K. E. Swick: On the boundedness and the stability of solutions of some non-autonomous differential equations of the third order. J. London Math. Soc., 44, 347-359 (1969).

[5] T. Yoshizawa: Stability Theory by Liapunov's Second Method. The Math. Soc. of Japan (1966). 